\title{
Assessment of cardiac time intervals by wavelet transform of the impedance cardiogram
}

\author{
Rodion Stepanov ${ }^{\mathrm{a}, *}$, Sergey Podtaev ${ }^{\mathrm{a}}$, Andrey Dumler ${ }^{\mathrm{a}, \mathrm{b}}$ and Sergey Chugainov ${ }^{\mathrm{a}, \mathrm{b}}$ \\ ${ }^{a}$ Institute of Continuous Media Mechanics, Perm, Russia \\ ${ }^{\mathrm{b}}$ Department of Introduction into Internal Diseases, Perm State Medical Academy, Perm, Russia
}

\begin{abstract}
.
BACKGROUND: Impedance cardiography (ICG) is an inexpensive, noninvasive technique for estimating hemodynamic parameters. ICG can be used to obtain the ejection fraction of the left atrium and to monitor systolic time intervals. Traditional ICG technique does not enable unambiguous detection of the left ventricle ejection time (LVET) and the time relationships between specific marker points.

OBJECTIVE: This work aims to approbate a new approach for ICG signal processing using wavelet transform (WT) and to investigate the possibilities of this approach for determination of the parameters which are related to the stroke volume (SV), in particular LVET.

METHODS: Thoracic tetrapolar polyrheocardiography method for simultaneous registration of ECG, ICG and phonocardiograms has been used. A control group consisted of eight healthy men aged 20-25 years. In addition, four patients with essential hypertension participated in the study. Wavelet representation of the ICG data produced local maxima in a two dimensional distribution of the wavelet coefficient. Each extremum point was characterized by the amplitude, scale and time, which determine SV.

RESULTS: LVET was defined as the scale corresponding to the E-wave maximum related to the systolic phase of the cardiac cycle. Also, we defined the initial systolic time interval (ISTI) as the time interval between R peak in the ECG and E-wave maximum on the wavelet plane. During functional test LVET and ISTI values defined by WT demonstrated a proper hemodynamic response to loading for the control group and patients with essential hypertension.
\end{abstract}

CONCLUSION: The proposed approach demonstrates the ability of ICG-WT technique for adequate assessment of SV parameters, including cardiac time intervals.

Keywords: Impedance cardiography, wavelet transform, hemodynamic parameters

\section{Introduction}

Impedance cardiography (ICG) is a inexpensive, noninvasive technique for assessment of hemodynamic parameters. ICG can be used to obtain left atrial ejection fraction and to monitor systolic time intervals. The principle underlying the rheography method is based on changes in the electrical resistance of a particular area of the body to a high-frequency alternating current proportional to blood volume variations in the area at any given time point in time. ICG can be used to obtain one of the key

${ }^{*}$ Corresponding author: Rodion Stepanov, Institute of Continuous Media Mechanics, Ak. Korolev str 1, 614013 Perm, Russia. Tel.: +73 422378394; E-mail: rodion@icmm.ru.

0928-7329/16/\$35.00 (c) 2016 - IOS Press and the authors. All rights reserved

This article is published online with Open Access and distributed under the terms of the Creative Commons Attribution NonCommercial License. 


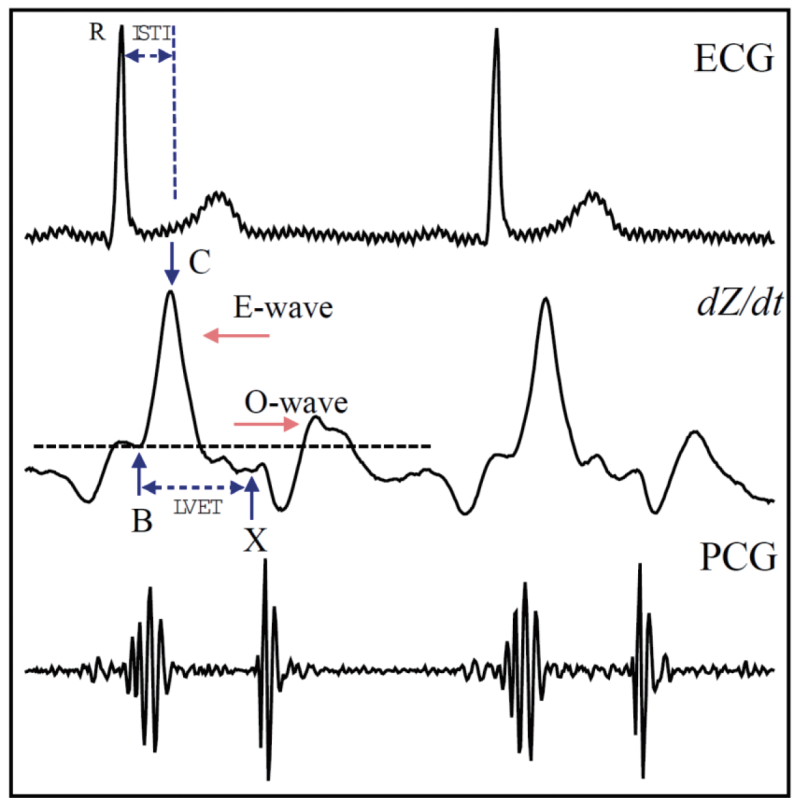

Fig. 1. Characteristic ECG, $d Z / d t$ and PCG signals, where B is the start of ejection of blood by the left ventricle, C is the major upward deflection occurring during systole, $\mathrm{O}$ is related to the diastolic phase, and $\mathrm{X}$ is the closure of the aortic valve.

hemodynamic parameters: stroke volume (SV). The cardiac dysfunctions can be detected by recognition of different ICG patterns. This allows one the sustain monitoring of hemodynamic parameters during screening diagnostics or intensive care. However further cardiac evaluation is needed. Doppler echocardiography [1] is a procedure that uses ultrasound technology to examine the heart or blood vessels, but it demands considerably less time and skills to produce accurate assessment.

There are several characteristic points which are usually considered to describe the ICG waveform [2]. These points are used to distinguish the physiological particularities of cardiac cycles. Figure 1 shows the so called $\mathrm{B}, \mathrm{X}, \mathrm{C}$ and $\mathrm{R}$ points, $\mathrm{E}$ and $\mathrm{O}$ waves.

The points $\mathrm{B}$ and $\mathrm{X}$ correspond to the first and second heart sounds. They are simultaneous with the maximal deflection at the apex and the closure of the aortic valve, respectively. In some cases the identification of the B point location is difficult. It may happen that the characteristic upstroke provides a marker of $\mathrm{B}$ point which is not sufficiently pronounced [3]. The $\mathrm{C}$ point is defined by the peak of $d Z / d t$ curve, which reflects the maximal derivative in the impedance. Ultrasound method measurements confirm that the $\mathrm{C}$ point may be related to the maximal velocity of the heart ejection [4]. For ICG the first maximum (i.e. E-wave) is related to the systolic phase of the cardiac cycle, and the second maximum with a smaller amplitude (O-wave) is related to the diastolic phase. The amplitude of the E-wave is proportional to the SV, and the amplitude of the O-wave correlates to a change in the volume of the left auricle during a short-term diastolic phase. In some cases the amplitude of the $\mathrm{O}$-wave proves to be an important diagnostic parameter [5,6]. Following the Kubicek formula [7], the stroke volume SV is proportional to the ejection time and the maximum value of the first derivative of the recorded impedance $Z(t)$ during the given cycle.

The left ventricle ejection time (LVET) is defined as the time interval of left ventricular ejection, which occurs between the opening of the aortic valve and its subsequent closure. However, as stated in some studies [8], there is evidence that ICG does not enable the detection of the onset of the aortic 
valve opening and closing process. Most of the ICG algorithms for LVET computation exhibit very low correlation coefficients and very high systematic estimation errors and dispersions [9]. The objective of this work is a demonstration of the wavelet transform for processing and interpretation of the impedance cardiogram waveforms and an investigation the possibilities of this approach to determine the parameters of the SV, in particular LVET. Also we measured initial systolic time interval (ISTI) - delay between electrical and mechanical pumping activities of the heart [10]. We note that ISTI is not particularly different from the electro-mechanical delay. We evaluated the ISTI and LVET depending on the heart rate in response to a postural test.

\section{Measurement and its processing algorithm}

\subsection{Measuring technique}

The control group was composed of eight healthy men aged 20-25 years. In addition, four patients with essential hypertension participated in the study. We used the method of computer thoracic tetrapolar polyrheocardiography for simultaneous registration ECG, ICG and phonocardiograms [11]. During the polyrheocardiogram registration done for six people (three subjects from the control group and three patients) the functional test (isometric load) was performed. Patients' legs were held raised at an angle of 30-40 degrees to the horizontal in order to create a static force. Another six people passed the thoracic tetrapolar polyrheocardiography without the functional test for comparison analysis.

\subsection{Wavelet transform}

The fundamental idea of wavelet transforms is that decomposition should be done with base functions which have a finite resolution in the physical and spectral spaces. Hence, the wavelet coefficients represent the local spectral characteristics of a signal. Continuous wavelet transform of the analyzing function $S(t)$ is given by convolution

$$
w_{S}(a, b)=\frac{1}{|a|} \int_{-\infty}^{\infty} S\left(t^{\prime}\right) \vartheta^{*}\left(\frac{t^{\prime}-b}{a}\right) d t^{\prime},
$$

where $w_{S}(a, b)$ is the wavelet coefficient of the scale $a$, and at the position $b$ and $\vartheta(t)$ it is the analyzing (mother) wavelet.

The function $S$ can be reconstructed using the inverse transform (see, e.g. [12])

$$
S(t)=\frac{1}{C_{\vartheta}} \int_{-\infty}^{\infty} \int_{-\infty}^{\infty} \vartheta\left(\frac{t^{\prime}-t}{a}\right) w_{S}\left(a, t^{\prime}\right) \frac{d a d t^{\prime}}{a^{2}} .
$$

The reconstruction Eq. (2) exists on condition that

$$
C_{\vartheta}=\frac{1}{2} \int_{-\infty}^{\infty} \frac{|\hat{\vartheta}(\omega)|^{2}}{|\omega|} d \omega<\infty
$$



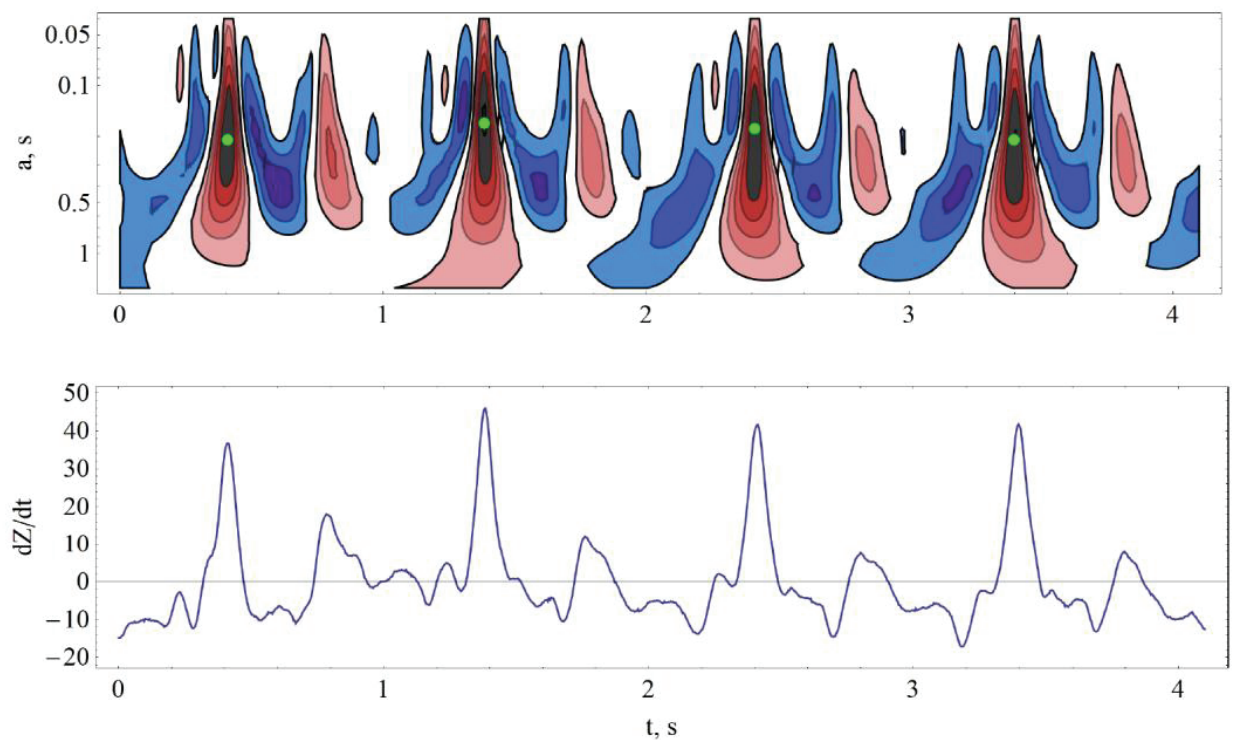

Fig. 2. 2D distribution of wavelet coefficients (top) and analysing signal (bottom). The red/blue colours denote positive/negatine values. The white area corresponds to the values of wavelet coefficients which are less than $10 \%$ of maximum value. The characteristic $\mathrm{C}$ points are shown by green points.

Here, $\hat{\vartheta}(\omega)=\int \vartheta(t) e^{-i \omega t} d t$ is the Fourier transform of the analyzing wavelet $\vartheta(t)$.

Let us define the wavelet transform of the analysing function $G(t)$, which is a derivation of the measured function $S(t)$, as follows

$$
w_{G}(a, b)=\frac{1}{|a|} \int_{-\infty}^{\infty} G\left(t^{\prime}\right) \vartheta^{*}\left(\frac{t^{\prime}-b}{a}\right) d t^{\prime} .
$$

Equation (4) can be differentiated by parts

$$
w_{G}(a, b)=\frac{1}{|a|} \int_{-\infty}^{\infty} S\left(t^{\prime}\right) \xi^{*}\left(\frac{t^{\prime}-b}{a}\right) d t^{\prime},
$$

where $\xi(t)=-\vartheta^{\prime}(t)$ is the differentiating wavelet. This means that wavelet transform of the analyzing function $G(t)$ can be obtained as the wavelet transform of the measured signal $S(t)$ using a new wavelet family $\xi(t)$. The advantage of the original wavelet differentiation algorithm [13] is a combination of filtering and differentiating procedures to process the rheocardiogram. We have chosen the so-called Mexican hat $\vartheta(t)=\left(1-t^{2}\right) \exp \left(-t^{2} / 2\right)$ as the analyzing wavelet, which provides high resolution in time in order to separate characteristic points (see Fig. 1). However the spectral resolution is better with the wavelet Morle $\vartheta(t)=\exp \left(-\frac{t^{2}}{2}+2 \pi i t\right)$, which is usually used for frequency filtering.

\section{Results}

The wavelet coefficients of the impedance derivative are shown in Fig. 2 as a two dimensional distribution. This distribution consists of alternated structures which correspond to local maxima and minima. 


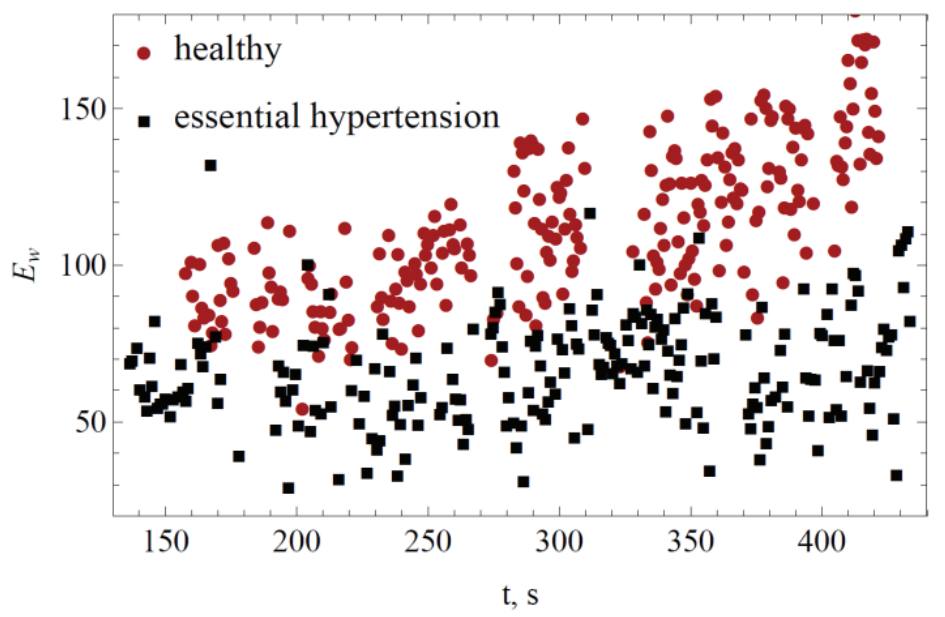

Fig. 3. Characteristic variations of the wavelet coefficient $E_{w}$ (amplitude of E-wave) during the postural test for healthy participants (red points) and for the patient with essential hypertension (black squares).

The most pronounced maxima (marked by points) represent E peaks in each cardiac cycle. Our method can be used to define the time moment $t_{E}$, the scale $a_{E}$ and amplitude $w_{E}$ for each extremum point (market by green point) in the 2D domain. It is considerably different from the analysis of an ordinal signal, which deals with the amplitude and time of the E-peak. The wavelet coefficient map gives three relevant parameters (instead of two) that are not affected by the noise and breath modulation of $Z$. The interpretation of these parameters is important to be justified.

First of all we consider an amplitude $w_{E}$. We have performed an isometric functional test (legs are lifted at an angle of 30-40 degrees to the horizontal) for verification conformity $w_{E}$ and stroke volume. The corresponding hemodynamic responses for two typical participants are shown in Fig. 3. For the healthy participant ( 25 year old man) the stroke volume increases simultaneously with a linearly increasing index $w_{E}$ that characterizes the maximal velocity of heart ejection. The amplitude $w_{E}$ restores its normal value within a few minutes after the end of the test. This behaviour accomplishes a proper hemodynamic response to loading known as an evidence of the Frank-Starling mechanism. Changes in the cardiac output were not found during the isometric stress for patients with the essential hypertension. A typical variation in $w_{E}$ is shown Fig. 3 for 55 year old man, who was given the following diagnosis: dilation of the left atrium and concentric left ventricular hypertrophy and coronary artery disease. Compensatory mechanisms did not work in this case because no direct adaptation to hemodynamic stress was observed.

Secondly, we check whether the scale $a_{w}$ of each extremum point with amplitude $E_{w}$ characterizes an extension of $\mathrm{E}$ wave in time. It can be used for estimation of LVET. Figure 4(a) shows that the wavelet estimate is well correlated with the RR intervals. The correlation coefficient calculated over 163 cardiac circles is 0.7 for healthy subjects and 0.6 for the patient with hypertension. Another subjects showed similar correlation in a range between 0.6 and 0.7 . We have also compared $a_{w}$ with a time interval between the first and second heart sounds. We have determined it using the phonogram recorded simultaneously with the impedance cardiogram.

The third parameter extracted from the E wave maximum is the initial systolic time interval. We define the ISTI as the time interval between the R peak in the electrocardiogram and the time $t_{E}$ of $E_{w}$ for each cardiac circle. ISTI is defined in healthy humans and the patient with hypertension at various heart rates. 


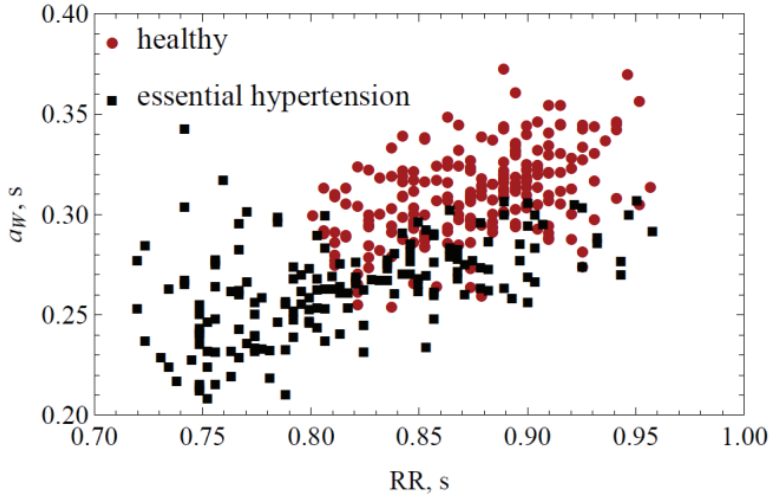

(a)

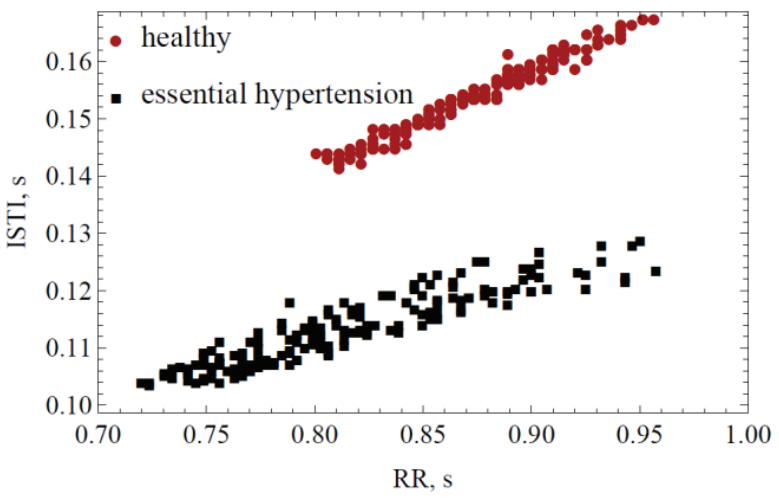

(b)

Fig. 4. Time and scale of wavelet $\mathrm{E}$ wave maximum for healthy subjects (red) and the patient with hypertension (black): $a_{w}$ (LVET estimate) vs RR intervals (a), ISTI vs RR interval (b).

Figure 4(b) shows a typical dependence of ISTI on the RR intervals. The absolute shortening of ISTI for the patient with hypertension can be attributed to compensatory reaction and increased myocardial contractility. Also we have observed the shortening of the ISTI with increasing heart rate in response to an exercise stimulus in the same spirit as in [10]. It is interesting that ISTI has different slopes for healthy subjects (ISTI $\propto 0.14 R R)$ and the patient with hypertension (ISTI $\propto 0.1 R R)$. Another tests demonstrate more scatter distribution of points than it is shown in Fig. 4(b). However taking into account the errors we have estimated the slope $0.15 \pm 0.1$ for healthy subjects and $0.1 \pm 0.3$ for the patient with hypertension. It is worthy of note that the variability of paraments for patients studied at rest (no load) is not enough to obtain dependencies shown in Figs 3 and 4.

\section{Conclusions}

Our results that are represented grapfically can serve as a demonstration of the capabilities of the method proposed. It is obvious that an additional statistical data is necessary for convincing conclusions. However, the proposed approach demonstrates that ICG-WT technologies can provide adequate assessment of cardiac time intervals, including LVET and ISTI. Our method can be used in clinical practice for early diagnostics of cardiovascular system remodelling in the course of different pathologies. It is shown that the wavelet transform is not only as a common tool for filtration, but it can also be used to study the corresponding time-frequency ICG structure defined by wavelet coefficients.

\section{Acknowledgment}

The work was supported by the Russian Science Foundation under project 14-15-00809.

\section{References}

[1] Bour J, Kellett J. Impedance cardiography - A rapid and cost-effective screening tool for cardiac disease [Review]. European journal of internal medicine. 2008; 19(6): 399-405. 
[2] Lababidi Z, Ehmke DA, Durnin RE, Leaverton PE, Lauer RM. The 1st derivative thoracic impedance cardiogram. Circulation. 1970; 41(4): 651-658.

[3] Lozano DL, Norman G, Knox D, Wood BL, Miller BD, Emery CF, et al. Where to B in dZ/dt. Psychophysiology. 2007; 44(1): 113-119.

[4] Kerkkamp H, Heethaar R. A comparison of bioimpedance and echocardiography in measuring systolic heart function in cardiac patients. In: Riu, PJ and Rosell, J and Bragos, R and Casas, O, editor. Electrical bioimpedance methods: applications to medicine and biotechnology. vol. 873 of Annals of the New York Academy of Sciences. Int Comm Promot Res Bio Impedance. New York Acad Sciences; 1999. p. 149-154.

[5] Schieken RM, Patel MR, Falsetti HL, Lauer RM. Effect Of Mitral Valvular Regurgitation On Trans-Thoracic Impedance Cardiogram. British Heart Journal. 1981; 45(2): 166-172.

[6] Pickett B, Buell J. Usefulness Of The Impedance Cardiogram To Reflect Left-Ventricular Diastolic Function. American Journal Of Cardiology. 1993 ; 71(12): 1099-1103.

[7] Kubicek WG, Karnegis JN, Patterson RP, Witsoe DA, Mattson RH. Development and evaluation of an impedance cardiac output system. Aerospace medicine. 1966; 37(12): 1208-1212.

[8] Ermishkin VV, Lukoshkova EV, Bersenev EY, Saidova MA, Shitov VN, Vinogradova OL, et al. Beat-by-beat changes in pre-ejection period during functional tests evaluated by impedance aortography: A step to a left ventricular contractility monitoring. IFMBE proceedings vol. 17; 2007. p. 655-658.

[9] Carvalho P, Paiva RP, Couceiro R, Henriques J, Antunes M, Quintal I., et al Comparison of systolic time interval measurement modalities for portable devices, Annual International Conference of the IEEE Engineering in Medicine and Biology, Buenos Aires Aug. 31 2010-Sept. 4 2010, p. 606âĂŞ609.

[10] Van Eijnatten MAJM, Van Rijssel MJ, Peters RJA, Verdaasdonk RM, Meijer JH. Comparison of cardiac time intervals between echocardiography and impedance cardiography at various heart rates. Journal of Electrical Bioimpedance. 2014; 5: 2-8.

[11] Zubarev M, Dumler A, Shutov V, Popov N. Assessment of left ventricular systolic function and diastolic time intervals by the bioimpedance polyrheocardiographic system. In: Riu, PJ and Rosell, J and Bragos, R and Casas, O, editor. Electrical Bioimpedance Methods: Applications To Medicine And Biotechnology. vol. 873 of Annals Of The New York Academy Of Sciences. New York Acad Sciences; 1999. p. 191-196.

[12] Daubechies I. Ten lectures on wavelets. Applied Mathematics. 1992; 61.

[13] Stepanov R, Frick P, Shukurov A, Sokoloff D. Wavelet tomography of the Galactic magnetic field. I. The method. Astronomy and Astrophysics. 2002; 391: 361-368. 